\title{
Application of Cluster Analysis on Gaussian-Mixture Probability Hypothesis Density Filter for Multiple Extended Target Tracking
}

\author{
Zhuo Cao, Xinxi Feng, Yinglei Cheng and Hongyan Li \\ Telecommunication Engineering Institute Air Force Engineering University, Xi’an 710077, China
}

\begin{abstract}
Based on the multiple Extended Target GaussianMixture Probability Hypothesis Density (GM-PHD) filter, a new algorithm of extended target track initiation and observation partition in the clutter environment are proposed. Firstly the paper take clustering trend of observation into account when carrying track initiation, which make the clustering results more convincing and increase computational efficiency; Then, the improved partition algorithm introduce the concepts of core distance and reached distance to save the sequence of measurement points and extract the measurement cluster. Simulation experiments show that the proposed initiation algorithm has a better computational cost over traditional algorithm when carrying track initiation. In the partition process, the new algorithm is not sensitive to the parameter selection and extended target measurement density, at the same time, the computational cost decreases.
\end{abstract}

Keywords-information fusion; extended target tracking; track initiation; measurement partition

\section{INTRODUCTION}

The traditional target tracking generally regards moving objects as single reflection point targets. In recent years, the technology of advanced sensor has made considerable progress, the modern sensors can detect a lot of reflection points on the surface of moving objects. So we call this type of target as extended target [1]. Random finite set theories [2] (RFS) set state and observation as units and operation process is simplified by a large margin. Mahler proposed the probability hypothesis density (PHD) filtering algorithm based on the random finite set theories, which opened up a new research direction for multiple target tracking. In 2009, Mahler applied it to the extended target tracking [3] and figured out the iterative updating formula. Followed by Granstrom[4], Gauss mixture technology is applied to the extended target PHD algorithm and the analytical solution was obtained.

Track initiation should be the primary problem for target tracking, the correctness is an effective measure to reduce the computational burden caused by combinatorial explosion. If the track initiation is not correct, it is impossible to carry on the target tracking. however, there are only few studies on the track initiation for extended target, the authors propose a method of extended target logical method to track initiation [5].

The observation partition is one of the key problems of extended target tracking filter. When tracking extended targets, one target may generate several reflection points, however, the sensor can't build the corresponding relation between measurements and targets reference [6] proposes a measurement partition method based on the distance classification, the algorithm is complex and it is difficult to partition the measurement sets effectively, reference [7] presents a K-means [8] algorithm to partition measurement sets, which has high computing efficiency, but the random selection of initial point will lead to different partition results and the algorithm can't partition measurement sets of arbitrary shape. The above methods are both difficult to effectively partition measurement sets when the measurement density distribution is uneven. The paper takes clustering tendency of measurement sets into account at the stage of track initiation firstly, Then we improve OPTICS algorithm [9] and use re-organization strategy to extract measurement sets.

\section{EXTEnded TARget Gauss MiXture Probability HYPOTHESIS DENSITY FILTER}

Extended Target Gaussian Mixture PHD (ET-GM-PHD) is a kind of approximate calculation method. Firstly we need to model the target motion equation and the observation equation. Two random finite sets $\boldsymbol{X}_{k}$ and $\boldsymbol{Z}_{k}$ are used to represent the k moment of the target state and the observation, that is

$$
\begin{gathered}
\boldsymbol{X}_{k}=\left\{\boldsymbol{x}_{k}^{1}, \boldsymbol{x}_{k}^{2}, \ldots, \boldsymbol{x}_{k}^{N_{x, k}}\right\} \\
\boldsymbol{Z}_{k}=\left\{\boldsymbol{z}_{k}^{1}, \boldsymbol{z}_{k}^{2}, \ldots, \boldsymbol{z}_{k}^{N_{z, k}}\right\}
\end{gathered}
$$

The state equation is expressed as

$$
\boldsymbol{x}_{k}^{i}=\boldsymbol{F}_{k-1} \boldsymbol{x}_{k-1}^{i}+\boldsymbol{G}_{k-1} \boldsymbol{\omega}_{k-1}^{i}
$$

In the formula, $\boldsymbol{F}_{k}$ represent state transition matrix, $\omega_{k}^{i}$ represents a zero mean Gauss white noise.

Measurement equation is 


$$
\boldsymbol{z}_{k}^{j}=\boldsymbol{H}_{k} \boldsymbol{x}_{k}^{j}+\boldsymbol{v}_{k-1}^{j}
$$

$\boldsymbol{H}_{k}$ represents measurement matrix, ${ }^{\boldsymbol{v}_{k}^{j}}$ represent a zero mean Gauss white noise with covariance of $\boldsymbol{R}_{k}^{j}$.

The predicted PHD can be expressed as

$$
D_{k \mid k-1}(\boldsymbol{x})=\sum_{j=1}^{J_{k \mid k-1}} \omega_{k \mid k-1}^{j} N\left(\boldsymbol{x}, \boldsymbol{m}_{k \mid k-1}^{j}, \boldsymbol{P}_{k \mid k-1}^{j}\right)
$$

In the formula, $J_{k \mid k-1}$ indicates the number of Gauss components, $\omega_{k \mid k-1}^{j}$ indicates that the weight of the Gauss components, ${ }^{\boldsymbol{m}_{k \mid k-1}^{j}}$ represents the predicted mean of the Gauss component, $\boldsymbol{P}_{k \mid k-1}^{j}$ indicates the covariance of the Gauss component.

The measurement update PHD can be expressed as

$$
D_{k \mid k}(\boldsymbol{x} \mid \boldsymbol{z})=L_{Z}(\boldsymbol{x}) D_{k \mid k-1}(\boldsymbol{x} \mid \boldsymbol{z})
$$

The likelihood function is in the expression of $L_{Z}(\boldsymbol{x})$

$$
\begin{aligned}
& L_{Z}(\boldsymbol{x}) \triangleq 1-\left(1-\mathrm{e}^{-\gamma(\boldsymbol{x})}\right) P_{D}(\boldsymbol{x}) D_{k \mid k-1}(\boldsymbol{x} \mid \boldsymbol{z})+ \\
& \mathrm{e}^{-\gamma(\boldsymbol{x})} P_{D}(\boldsymbol{x}) \sum_{\boldsymbol{P} \angle \boldsymbol{Z}_{k}} \omega_{P} \sum_{\boldsymbol{W} \in \boldsymbol{P}} \frac{\gamma(\boldsymbol{x})^{|\boldsymbol{W}|}}{d w} \cdot \prod_{Z_{k} \in W} \frac{\phi_{z}(\boldsymbol{x})}{\lambda_{k} c_{k}\left(\boldsymbol{z}_{k}\right)}
\end{aligned}
$$

The mean value of the number of clutter measurement is in the expression of $\lambda_{k} \triangleq \beta_{F A, k} V_{s}$, the spatial distribution of the clutter is in the expression of $c_{k}\left(\boldsymbol{z}_{k}\right)$ and $\boldsymbol{p} \angle \boldsymbol{Z}_{k}$ represent all the possible division of the measurement set.

\section{EXTENDED TARGET TRACK INITIATION}

At each moment, the extended target generates a number of measurements, so it can not directly use the point target track initiation method. According to the research of traditional multi target track initiation has been more mature, so the existing methods of solving multiple extended targets track initiation adopt some traditional track initiation methods, We determine whether the centroid state of the expansion meet track initiation conditions, and then make the track confirmation.

However, the spatial clustering operation can get a clustering result, this may bring two direct consequences: a) If the measurement data are random, the clustering results can't be explained, and it is also useless.

b) The lack of a priori knowledge about the measurement set, which brings great difficulties to the selection of algorithms and parameters.

It is assumed that the measurement of clutter is random in space, so it can be considered that the measurement of clutter isn't clustered, thus the aggregation of measurement is largely derived from the extended target. In order to save the limited computing resources and more effectively understand the measurement data, it's necessary to analyze the clustering trend [10] of measurement data before clustering operation firstly.

In the track initiation stage, the clustering trend analysis can bring two benefits:

a) Without clustering towards clutter measurement and taking multiple assumptions through centroid prediction to identify possible track, which reduce the false track probability and calculation cost significantly.

b) Analyzing clustering trend before doing clustering operation can reduce the possibility of imposing the error clustering structure on the measurement and make the clustering results more accountable.

The initiation implementation steps are shown in figure I:

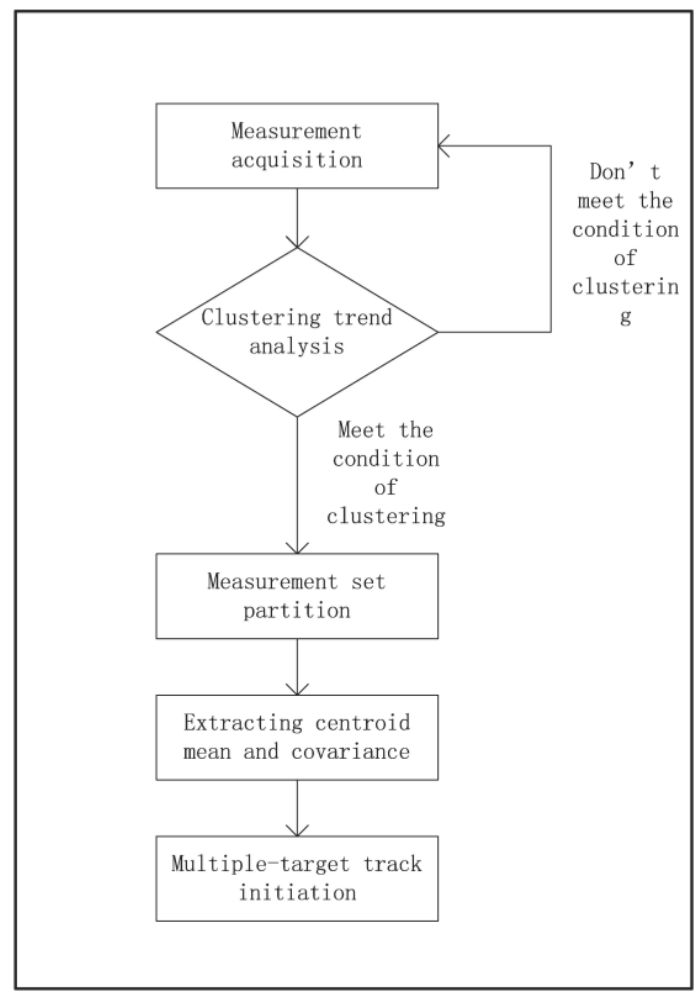

FIGURE I. THE FLOW CHART OF EXTENDED TARGET TRACK INITIATION

In this paper, we take the Nearest Neighbor Index(NNI) method to analyze the clustering trend of the measurement. The nearest neighbor index NNI is expressed as 


$$
N N I=\frac{d(N N)}{d(\text { ran })}=\frac{\sum_{i=1}^{N} d_{i}^{\min } / N}{d(\text { ran })}
$$

In the formula, $d_{i}^{\text {min }}$ represents the distance of the nearest neighbor measurement; $N$ represents the number of measurement; $d(\mathrm{ran})$ represents the average nearest neighbor distance of random distribution and we set it as $d($ ran $)=0.5 \sqrt{A / N}$, in the formula $A$ represent the measurement area. When $N N I<1$, the measurement should be an aggregation distribution, When $N N I=1$, the measurement should be a random distribution, When $N N I>1$, the measurement should be an uniform distribution.

\section{Extended TARget Measurement Partition}

Accurate filter of PHD need to take into account all the partition of the measurement, the number of measurement partition will increase sharply with the number of measurement increase, thus it is difficult to achieve in practical application. So how to partition the measurement effectively play an important role in the multiple extended target filter.

For the Gauss mixture probability hypothesis density filter, the cluster algorithm [11] based on density is the best one, and the OPTICS algorithm is a kind of density based algorithm. The algorithm is not sensitive to noise and adopt density ordering strategy, which establish the concept of core distance and reached distance to find arbitrary shape and different density extended target measurement.

This paper designs an improved algorithm based on the result redistribution strategy. The new algorithm assumes that the smaller the distance between two measurement points, the greater probability of them belong to the same extended target. For this purpose, we add a selection queue for each measurement point, which is used to associate the nearest neighbor measurement point.

\section{SiMUlation EXPERIMENT AND ANALYSIS}

\section{A. Experimental parameter setting}

Considering the motion of single sensor tracking four targets in the two-dimensional space, a two-dimensional observation area for $\mathrm{x} \sim[-1000 \mathrm{~m}, 1000 \mathrm{~m}], \mathrm{y} \sim[-1000 \mathrm{~m}, 1000 \mathrm{~m}]$, the sensor coordinate $S=(1000 \mathrm{~m}, 0 \mathrm{~m})$, the sampling period is $\mathrm{T}=1 \mathrm{~s}$, the initial state, birth and death time are shown in Table 1. The measurement last for 200s. 1-100s, the extended target does not appear and the measurement space consist of clutter measurement; 101 -200s, 4 extended targets have emerged step by step. We achieved 200 times Monte-Carlo simulation.
TABLE I. INITIAL STATE AND TRACKING OF TARGETS

\begin{tabular}{|c|c|c|}
\hline \multirow{2}{*}{ Target } & \multicolumn{2}{|c|}{ Initial state and tracking of targets } \\
\cline { 2 - 3 } & Initial state & Born/Death time \\
\hline Target 1 & {$[100400 / 99450-1350 / 99]$} & $101 / 200 \mathrm{~s}$ \\
\hline Target 2 & {$[-4501050 / 50-450-250 / 50]$} & $150 / 200 \mathrm{~s}$ \\
\hline Target 3 & {$[450-700 / 33-209-591 / 33]$} & $170 / 200 \mathrm{~s}$ \\
\hline Target 4 & {$[-350550 / 33-350850 / 33]$} & $170 / 200 \mathrm{~s}$ \\
\hline
\end{tabular}

Extended target state model as:

$$
\boldsymbol{x}_{k}=\left[\begin{array}{llll}
1 & 0 & T & 0 \\
0 & 1 & 0 & T \\
0 & 0 & 1 & 0 \\
0 & 0 & 0 & 1
\end{array}\right] \boldsymbol{x}_{k-1}+\left[\begin{array}{cc}
T^{2} / 2 & 0 \\
0 & T^{2} / 2 \\
T & 0 \\
0 & T
\end{array}\right]\left[\begin{array}{c}
\omega_{1, K} \\
\omega_{2, K}
\end{array}\right]
$$

In the formula, $x_{k}, y_{k}$ represents the position coordinate of the target, $v_{x} 、 v_{y}$ represents the velocity components respectively. The process noise $\left[\omega_{1, k}, \omega_{2, k}\right]^{\mathrm{T}}$ follows a zero mean Gauss distribution, the PHD for the new birth target is

$$
\begin{gathered}
D_{b}(\boldsymbol{x})=0.1 \times N\left(\boldsymbol{x} ; \boldsymbol{m}_{b}, \boldsymbol{P}_{b}\right)+0.1 \times N\left(\boldsymbol{x} ;-\boldsymbol{m}_{b}, \boldsymbol{P}_{b}\right) \\
\boldsymbol{m}_{b}=[250,0,250,0]^{\mathrm{T}} \\
\boldsymbol{P}_{b}=\operatorname{diag}(100,25,100,25)
\end{gathered}
$$

The measurement equation is

$$
\boldsymbol{Z}_{k}=\left[\begin{array}{cccc}
1 & 0 & 0 & 0 \\
0 & 1 & 0 & 0
\end{array}\right] \boldsymbol{x}_{k}+\left[\begin{array}{l}
R_{1, k} \\
R_{2, k}
\end{array}\right]
$$

In the formula, the clutter $\left[R_{1, k}, R_{2, k}\right]^{\mathrm{T}}$ follows a zero mean Gauss distribution, and its component standard deviation is $\sigma_{\mathrm{R} 1}=\sigma_{\mathrm{R} 2}=20 \mathrm{~m}$. The target survival probability is $\mathrm{P}_{\mathrm{s}}=0.98$, the detection probability is $P_{d}=0.98$. the number of target measurement generate with mean of $10,20,40,80$ respectively, and follows an average distribution in the measurement square. The number of clutter follows a Poisson distribution with mean of 30. The maximum Gauss component number is $J_{\max }=100$, the pruning threshold is $T_{\mathrm{P}}=10^{-4}$, the combined threshold is $U=4$, the domain radius is $\varepsilon=30$, the minimum measurement number is MinPts $=6$. 


\section{B. Simulation results and analysis}

We analyze the clustering tendency of measurement from $1 \mathrm{~s}$ to 100 s and results show that the observation doesn't meet the requirement to be clustered, thus there's no need for later track initiation process and the false track initiation probability decrease. As shown in Figure II, compare the proposed algorithm in this paper with the algorithm proposed in reference, the running time cost of the new algorithm accounts for merely $26 \%$ of the extended target logic track initiation algorithm on the condition that ensuring higher track probability and lower false track probability. Computational efficiency has been significantly improved.

In order to compare the computational efficiency and effectiveness of the proposed algorithm with the traditional partition algorithm . In Figure III, the real target track and all the measurements are given.

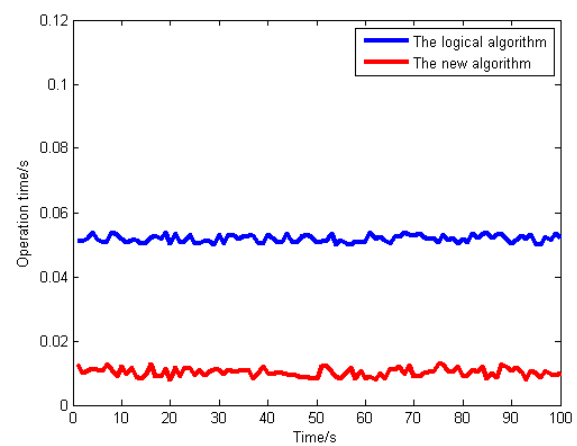

FIGURE II. COMPARISON OF TRACK INITIATION OPERATION TIME

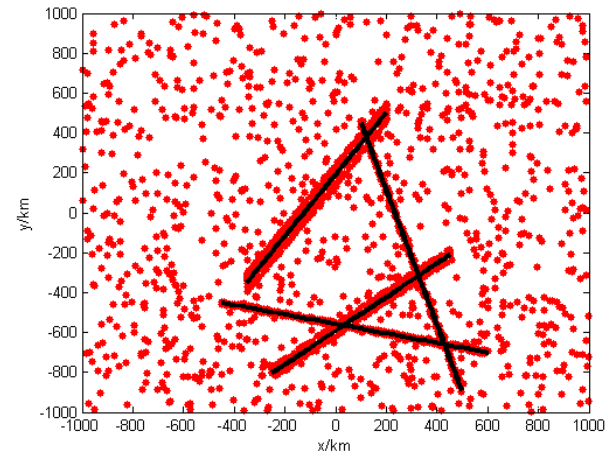

FIGURE III. REAL TRACKS AND MEASUREMENTS

It can be seen from figure IV, large deviation generated by distance partition algorithm and DBSCAN partition algorithm in reference [12] on the number of targets. and the proposed partition algorithm has filter the clutte, thus target numbers can be estimated accurately.

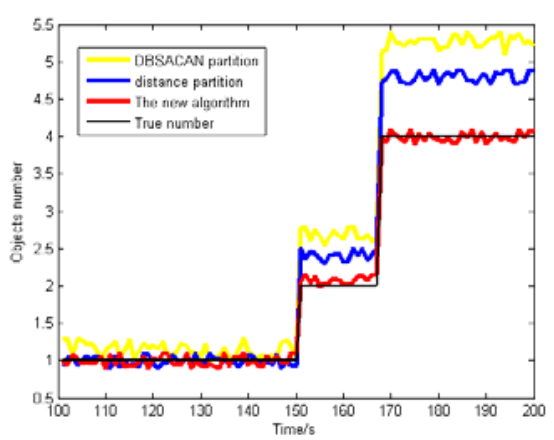

FIGURE IV. TARGETS NUMBER ESTIMATING

We use Optimal Sub Pattern Assignment (OSPA) distance [13] to evaluate the performance of different algorithms, the OSPA distance is defined as follows

$$
d_{p}^{c}(X, Z)=\left(\frac{1}{n}\left(\min _{\pi \in \Pi_{n}} \sum_{i=1}^{m} d^{(c)}\left(x_{i}, z_{\pi_{(i)}}\right)^{P}+c^{p}(n-m)\right)\right)^{1 / p}
$$

The selection of the parameters $\mathrm{p}$ and $\mathrm{C}$ has influence on the estimation deviation of target number and position coordinate. In the simulation experiment, we set the parameters $\mathrm{p}=2, \mathrm{c}=80$. FigureV dedicates the OSPA distance comparison of Monte Carlo simulation experiment, we can see that the classification accuracy of proposed algorithm is significantly better than the traditional algorithm. When the number of extended targets is changed, the curve of OSPA distance towards different algorithm is larger at the same time.

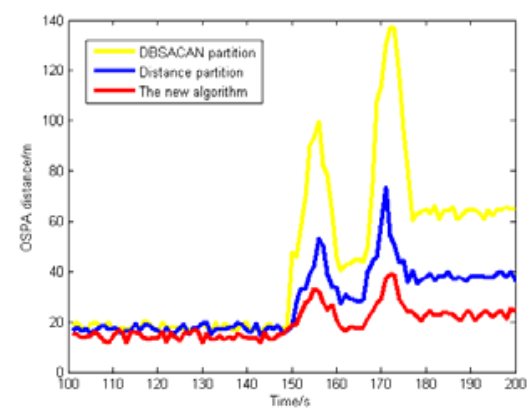

FIGURE V. OSPA DISTANCE

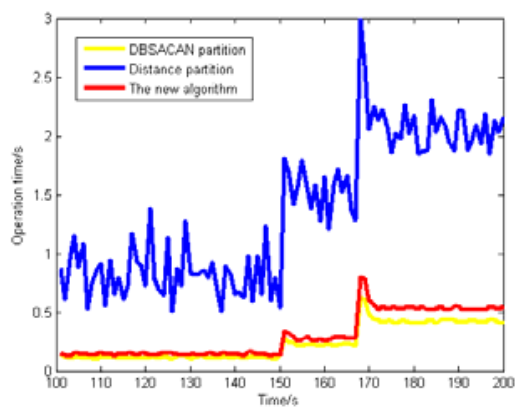

FIGURE VI. OPERATION TIME OF MEASUREMENT PARTITION 
Figure VI. gives three kinds of partition algorithm in the average running time comparison, the proposed algorithm can partition the measurement and filter the clutter measurement at the same time, we can conclude that the proposed algorithm in computational efficiency is much better than the distance partition algorithm, compared with DBSCAN partition algorithm, the operation time of proposed algorithm has a slight increase.

Responding to the problems of observation partition and extended target track initiation in the clutter environment, first of all, the paper introduce clustering trend analysis into track initiation process and propose a track initiation algorithm based on clustering trend analysis, the new algorithm can reduce the error initiation possibility by avoiding the error clustering structure imposed on the clutter measurement and computational efficiency is significantly improved; In the process of measurement partition, the proposed algorithm is applicable for the extended target of different density and shape can achieve better classification, clutter filtering properties of the algorithm itself has the same effect in ensuring the division significantly reduced the computation, is not sensitive to the choice of parameters also makes the algorithm robust strong.

\section{REFERENCES}

[1] Liu M Q, Lan J. Advance theory and application of target tracking[M]. Beijing: Science Press, 2015.202-203.

[2] Mahler R P S. Multitarget Bayes filtering via first-order multitarget moments[J]. IEEE Transactions on Aerospace and Electronic Systems, 2003, 39(4): 1152-1178.

[3] Mahler R. PHD filters for nonstandard targets, I: extended targets [C]. 12th International Conference on Information Fusion, Seattle, WA, USA, July, 2009, 915-921.

[4] Granstrom K, Lundquist C, and Orguner O. Extended target tracking using a Gaussian-mixture PHD filter[J]. IEEE Transactions on Aerospace and Electronic Systems, 2012, 48(4): 3268-3286.

[5] Han Y L, Zhu H Y, Han C Z. A Multi-Target Filter Based on Random Matrix[J]. Journal of Xi' an Jiao Tong University, 2015, 49(7): 98-104.

[6] Granstrom K, Lundquist C, and Orguner O. A Guassisan mixture PHD filter for Extended target tracking[C]. Proceedings of the 13th International Conference on Information Fusion, Edinburgh, UK, 2010: $1-8$.

[7] Li Y X, Xiao H T, Song Z Y, Hu R, Fan H Q. A new multiple extended target tracking algorithm using PHD filter[J]. Signal processing, 2013, 93: 3578-3588.

[8] Deng M, Liu Q L, Li G Q, Huang J B. Spatial clustering analysis and its application[M]. Beijing: Science Press, 2011.43-49.

[9] ANKERST M, BREUNIG M M, KRIEGEL H P, et al. OPTICS: ordering points to identify the clustering structure [J]. ACM SIGMOD Record, 1999, 28(2) : 49-60.

[10] BANERJEE A, DAVERN. Validating clusters using the Hopkins statistic[C]. Proc of IEEE International Conference on Fuzzy Systems Piscataway. IEEE Press, 2004: 1749.

[11] Tao Pei, Ajay Jasra, David J. Hand, A.-Xing Zhu, Chenghu Zhou. DECODE: a new method for discovering clusters of different densities in spatial data[J]. Data Mining and Knowledge Discovery, 2009, (3): 337-369.

[12] Ristic B, Vo B N, Clark D, Vo B T. A metric for performance evaluation of multi-target tracking algorithms. IEEE Trans. Signal Process. 2011, 59, 3452-3457.

[13] Ristic B, Vo B N, Clark D, Vo B T. A metric for performance evaluation of multi-target tracking algorithms. IEEE Trans. Signal Process. 2011, 59,3452-345. 